\title{
Gradhiva
}

GRADHI

Revue d'anthropologie et d'histoire des arts

32 | 2021

Livres sorciers

\section{Palimpseste. L'écriture rituelle au second degré}

\section{Andrea-Luz Gutierrez-Choquevilca}

\section{OpenEdition}

Journals

Édition électronique

URL : https://journals.openedition.org/gradhiva/5306

DOI : $10.4000 /$ gradhiva.5306

ISSN : 1760-849X

\section{Éditeur}

Musée du quai Branly Jacques Chirac

\section{Édition imprimée}

Date de publication : 24 mars 2021

Pagination : 10-23

ISBN : 978-2-35744-132-3

ISSN : 0764-8928

Référence électronique

Andrea-Luz Gutierrez-Choquevilca, « Palimpseste. L'écriture rituelle au second degré », Gradhiva [En ligne], 32 | 2021, mis en ligne le 02 avril 2021, consulté le 25 mai 2021. URL : http:// journals.openedition.org/gradhiva/5306 ; DOI : https://doi.org/10.4000/gradhiva.5306 
nummonis

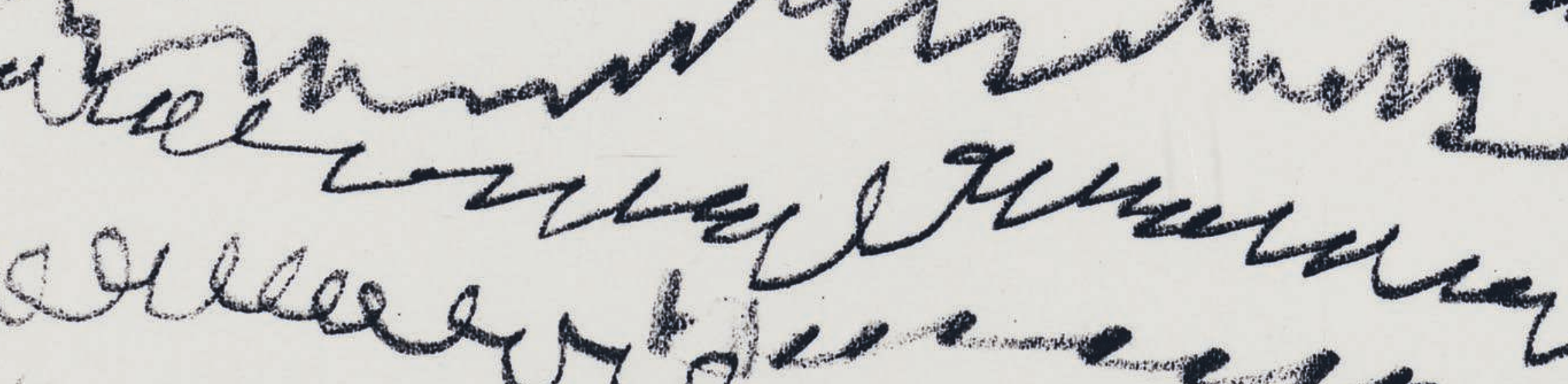
reaterinis abrunum retorna coureceroidh veru@3lernu 
Introduction

Palimpseste

Lécriture rituelle

au second degré

\section{Andrea-Luz \\ Gutierrez-Choquevilca}


1. Une prolixe littérature est consacrée à la question de l'alphabétisation

Le phénomène de l'écriture

efficace reste un domaine

encore peu exploré. Parmi

les exceptions, voir: Glassner

2008; Déléage 2013 et 2017;

Koch Piettre et Batsch (dir.)

2010; Davies 2009; Wogan

1994; Rendu-Loisel 2018;

Frai,

Guillaume-Pey 2016.

\section{LE LIVRE INFINI}

Il me dit que son livre s'appelait le livre de sable, parce que ni son livre ni le sable n'ont de commencement ni de fin.

Borges 1978: 100

Aucune des pages de ce livre ne peut être lue deux fois, car ce livre est infini. Il contient tous les livres possibles, passés et à venir. Il n'a par conséquent ni de commencement ni de fin. Cette révélation laisse place à la stupeur du personnage borgésien à qui est remis le livre de sable. Si les textes contenus dans ce livre varient à chaque lecture, si la signification des mots change au-delà de l'intention de son ou de ses auteurs, il est possible que ce livre contienne à lui seul toutes les lectures et tous les déchiffrements. Il exprimerait alors l'utopie, celle d'une écriture qui ferait corps avec l'expérience infinie du temps, de l'espace, du monde. L'expérience d'un «livre univers». L'écrivain argentin Jorge Luis Borges développe cette utopie dans une nouvelle décrivant la bibliothèque hexagonale de Babel, celle qui contient «tout ce qu'il est possible d'exprimer dans toutes les langues», qui inclut le «récit véridique de ta mort» ou «l'éclaircissement des mystères fondamentaux de l'humanité » (Borges 1983 : 127-129). Dans cette fiévreuse bibliothèque, les hasardeux volumes courent le risque incessant de se muer en d'autres. Sorcier, le livre ne l'est que pour celui qui nourrit le désir de s'emparer de l'infini dans sa course - toutes les signnifications possibles -, de réduire la pensée au format in-folio, tels ces inquisiteurs de la bibliothèque imaǵinaire de Babel «habités par le délire de conquérir les livres chimériques de l'Hexagone cramoisi : livres de format réduit, tout-puissants et maǵiques » (ibid.: 132).

Le caractère infini dont témoigne la fiction de Borges fait écho aux variations sur les écritures magiques étudiées dans le présent volume. Le lecteur découvrira ici autant de livres imprimés, inscrits de textes ou d'images, que de manuscrits anciens ou modernes, de documents officiels ou d'artefacts rituels à inscriptions. D'un point de vue formel, le travail de composition de ces textes n'est pas étranger à celui de l'écrivain en littérature, qui procède par retraits, appendices de textes possibles, pas encore définis, en puissance ou à venir sur les espaces restés blancs de la page. Ces pages où se jouxtent les paperoles de Marcel Proust illustrent un geste analoguue, procédant par ajouts et superpositions de matières et de siǵnes, allant jusqu'à saturer les limites et la linéarité du support, comme celles de la mémoire et du temps. La diversité des matières et supports sur lesquels le signne est inscrit est analoǵue à la variabilité des formes sémiotiques qu'il revêt en tant que symbole, icône ou index, entretenant un rapport toujours complexe avec l'oralité. L'écrit fait signe vers la parole vive qui le précède ou lui succède, dans l'acte de lecture et de déchiffrement. Il est la trace d'une image mentale, d'un rite, d'une oralisation passée ou à venir. Il renferme enfin un savoir possible et une parole virtuellement énoncée, tel un commutateur entre plusieurs plans de réalité autrement séparés.
Ce livre sur le livre propose une analyse anthropologique de l'usage magique ou performatif de l'écriture à travers ses variations dans l'espace et dans le temps. Il réunit les contributions d'anthropologues, de philologues, d'archéologues ou d'historiens ayant mené leur enquête en Europe, en Amérique et en Afrique. À la différence de l'usage dévolu aux écritures ordinaires, vouées à laisser la trace d'une pensée dont l'auteur est absent, l'usage extraordinaire de l'écriture se singularise par son ancrage dans une situation d'échange immédiat et extraordinaire entre les humains, les esprits et les dieux. Sa vocation première n'est pas de stocker un savoir menacé par l'oubli, mais de transformer l'ordre du monde et les sujets qui l'habitent. Posant un regard critique sur le «grand partage» opposant la raison graphique à la pensée sauvage, l'anthropologie des écritures rituelles examine l'étrange pouvoir prêté au texte de transformer la matière sous certaines conditions. Elle met au jour des graphies subversives et des représentations du livre magique régies par une logique du concret, plutôt que par des conventions stables de lecture et d'écriture. Comment décrire la nature sémiotique hybride de ces textes extraordinaires? Que nous enseiǵne-t-elle sur le pouvoir du signe écrit? L'écrit magique est-il au texte conventionnel ce que la parole rituelle est à l'énonciation ordinaire? Une manifestation du langage agissant, libéré des conventions de la notation textuelle? Pour comprendre les enjeux praǵmatiques d'un usage rituel de l'écriture, il faut repartir du commencement. Explorer l'acte d'inscription, le geste du tracé et du déchiffrement, les savoirs et l'expérience qui accompagnent l'avènement de ces textes. La question de l'invention de l'écriture et les hypothèses fonctionnalistes qui abondent dans la littérature n'intéresseront pas directement les contributeurs de ce volume ${ }^{1}$. L'attention porte ici sur l'efficacité prêtée au texte et sur la manière dont les sociétés humaines se sont appropriées la maǵie de l'écriture.

\section{LIAISON MAGIQUE}

S'il ouvre à certains les portes du paradis, le texte maǵique implique une transformation radicale de la relation du sujet au langage et à l'existant. C'est moins la signification que l'effet produit qui intéresse les usagers de ces artefacts graphiques. Pris dans l'enǵrenage d'un réseau d'acteurs, de scribes, de lecteurs et de récipiendaires invisibles, l'écrit magique acquiert son autonomie propre, matérielle et idéelle: aǵissant à la manière d'un index, d'une icône ou d'un symbole, il permet aux usagers de convoquer des êtres dont l'intercession est requise dans les affaires humaines pour préserver ou ôter la vie, lier des êtres en scellant un pacte d'amour, annoncer la fortune, transmettre un messaǵe aux dieux et accompaǵner les âmes au ciel. L'écriture est un instrument privilégié de communication entre les humains et le monde invisible. La liaison épistolaire qui se tisse entre les vivants et les morts dans l'Égypte ancienne est sur ce point exemplaire. Des «lettres aux morts» écrites en tachyǵraphie hiératique sur divers supports (papyrus, vaisselles en 
Palimpseste, l'écriture rituelle au second degré

Andrea-Luz Gutierrez-Choquevilca

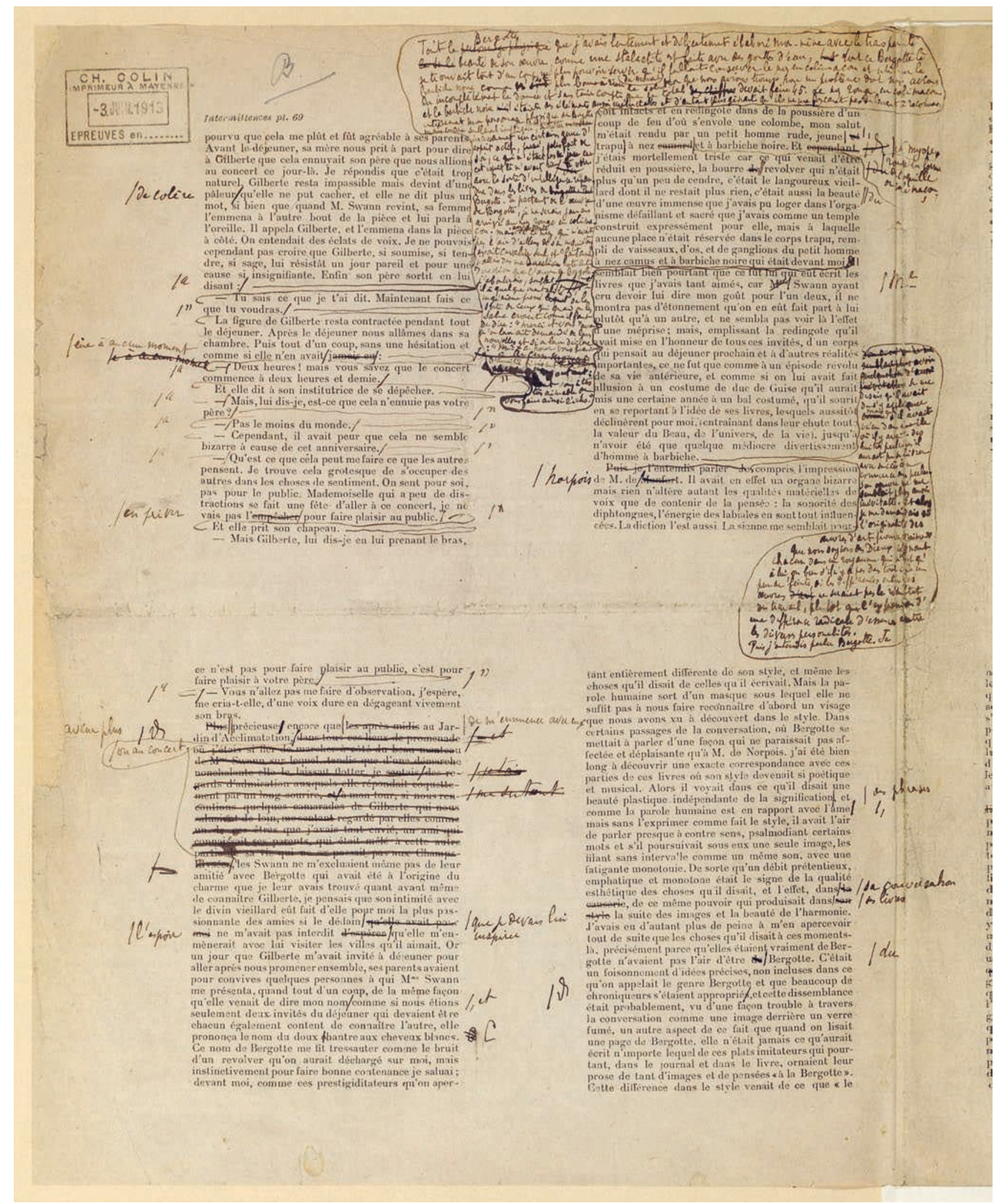

Marcel Proust, Placards corrigés pour Du côté de chez Swann, 1913

Paris, Bibliothèque nationale de France (BnF), NAF 16753 Folio 50 verso. Photo @ BnF, Dist. RMN-Grand Palais / image BnF. 

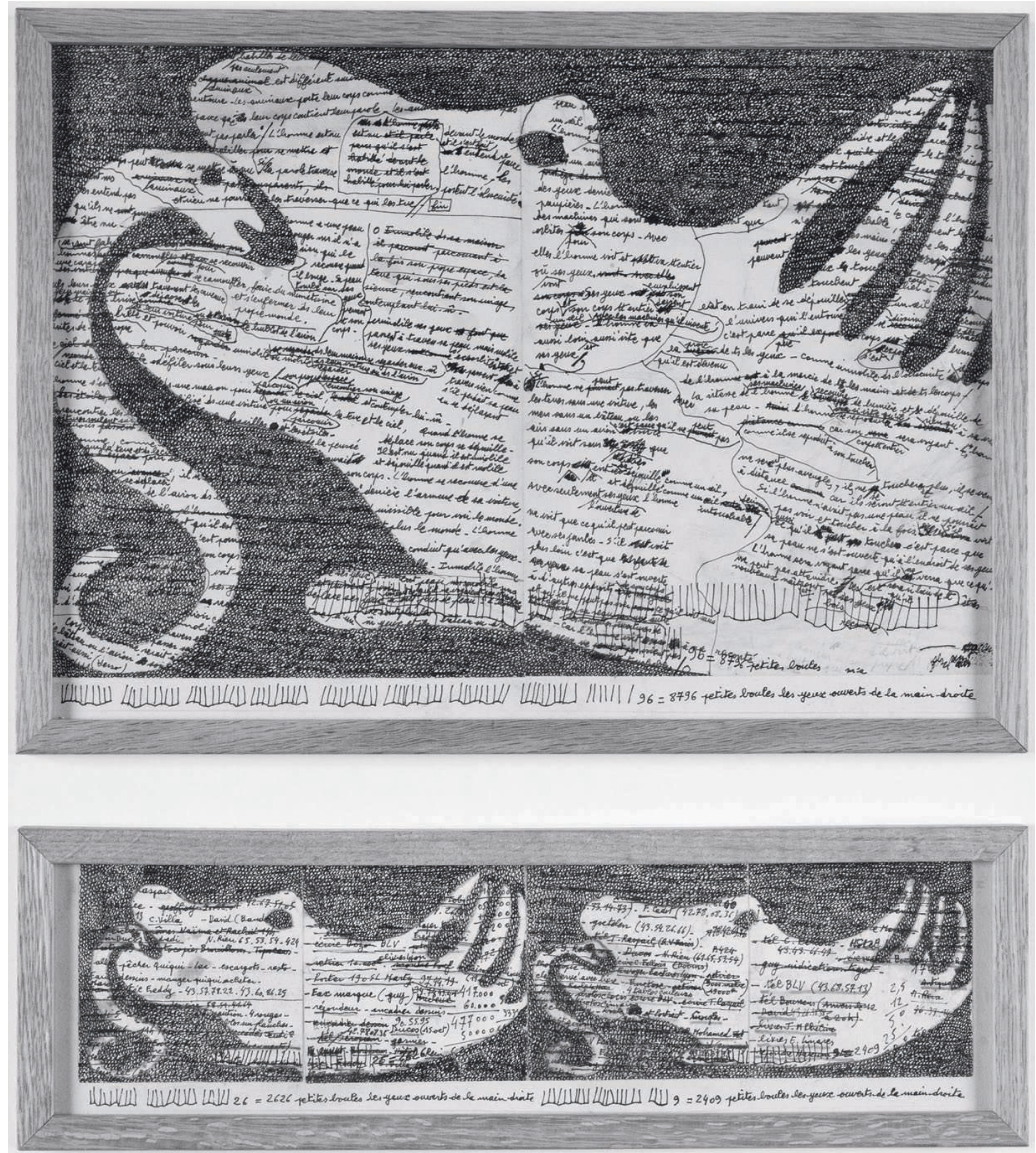

Jean-Luc Parant, Série Machines à voir. Les yeux goinfres: les mangeurs de textes. Brouillons brouillés, 1992

Encre de chine, crayon de couleur et crayon sur papier. Fonds national d'art contemporain. Acquisition en 1993. Attribution au Musée national d'art moderne, Centre Pompidou. Photo @ Centre Pompidou, MNAM-CCI, Dist. RMN-Grand Palais / Philippe Migeat @ Paris, ADAGP, 2021. 
céramique, pièce de lin, le revers d'une stèle), attestées de la fin de l'Ancien Empire (vers 2300 av. notre ère) jusqu'au VII ${ }^{\mathrm{e}}$ siècle av. notre ère, matérialisent à la fois une parole adressée et une relation contractuelle qui s'établit entre les vivants et les morts (Sylvie Donnat, ce volume). Le destinataire invisible de ces lettres est un défunt ǵlorifié, bénéficiaire des rites sakhou, dont il est attendu qu'il se montre favorable envers ses héritiers. Une parole humaine est ainsi transmuée vers le monde des morts, dans l'espoir d'influer sur les décisions qui y seront prises. Un aspect remarquable de ces écritures est leur dimension dialogique. Supports d'une parole virtuellement énoncée, certains objets votifs, comme le bol de Berlin, dévoilent sur un même espace scriptural la voix des défunts et celle des vivants. Ils offrent ainsi au scripteur la possibilité d'introduire un acte d'interpellation. De la période ramesside (1300-1070 av. n. è.) au Nouvel Empire, les amulettes pharaoniques, petits billets de papyrus pliés, inscrits d'une formule dans l'écriture manuscrite «hiératique» attestent quant à elles d'un processus inverse, qui met en place une transmutation de la parole des dieux parmi les vivants. L'écrit se réfère à un hypotexte issu d'un savoir lettré spécialisé. L'usage des amulettes se distingue cependant par une praǵmatique spécifique, fondée sur la contiguïté intime de ces objets avec le corps des usagers. L'écrit devient une sorte d'appendice. Il est le support d'une agentivité acquise par délégation, à travers la figure du ritualiste qui joue le rôle de médiateur dans la mobilisation d'une puissance divine. Le pouvoir conféré à l'écrit est inséparable d'une parole rituelle énoncée. Son efficacité découlet-elle pour autant de sa visibilité en tant que texte? Un dénominateur commun entre les amulettes talismaniques à écritures de l'Égypte ancienne et celles, plus contemporaines et d'origine islamique, découvertes dans la décharǵe de Mbebeuss à Dakar est que leur efficacité ne dépend précisément pas du caractère ostensible du siǵne linguistique, puisque ce dernier peut aussi bien rester dissimulé au regard (voir l'entretien avec Alain Epelboin et Jean-Charles Coulon dans ce volume). Il en va ainsi des écritures présentes sur des tuniques talismaniques et des ceintures à boucles contenant des textes, présentés dans la collection d'AlEp. La fermeture d'une amulette ou le camouflage de son texte dissimule au regard le cœur de son dispositif graphique, créant une dialectique entre un intérieur et un extérieur, faisant de l'artefact dans son ensemble une puissance exhibant un pouvoir divin dissimulé. L'invisibilisation de l'écrit repéré dans l'usage des amulettes à textes a-t-elle pour effet de redoubler le mode de manifestation privilégié des dieux, sous la forme d'une absence révélée ou d'une présence invisible? C'est le pouvoir que possède l'écriture de rendre visible un signe ou un être absent qui est en jeu ou fait l'objet d'un déplacement. Certains textes vont jusqu'à s'affranchir de toute matérialité graphique, comme la bibliothèque imaginaire de livres virtuels dont témoignnent les chamanes amérindiens (AndreaLuz Gutierrez-Choquevilca, ce volume; Déléage 2017). La fonction de l'écrit ne se réduit guère à une simple technique de communication à distance. Une fois insérée dans un dispositif rituel, elle devient l'indice et la manifestation sensible d'une présence dévoilée. Si en tant qu'icône elle peut être appréhendée dans sa dimension esthétique, en tant qu'index, sa lisibilité immédiate n'est pas requise pour fonder son efficacité.

\section{L'ÉGRITURE AU SEGOND DEGRÉ}

Tel un parchemin dont le texte primitif n'a pas été entièrement effacé, dont la trace subsiste encore mais sur lequel est venu se superposer un nouveau texte, inspiré ou non du premier, l'écrit magique doit être lu à la croisée d'intentions multiples, parfois contradictoires. Il est le texte d'un texte, le signe d'un signe et se caractérise par une réflexivité du langage. Un premier niveau de «recyclaǵe» du signe linguistique apparaît dans la conversion entre imaǵe et symbole repérée sur le texte (Déléage et Morin 2018). Il n'est pas sans évoquer la forme expressive libératrice contemporaine de l'art brut. Dans l'œuvre-monde d'Adolf Wölfli ou de Jean Luc Parant ${ }^{2}$, la révélation de messages invisibles transparaît sur des fresques saturant l'espace visuel, mêlant des symboles écrits savamment ordonnés, des partitions musicales et des figures corporéiformes dessinant des sortes de mélodies visuelles. Le symbole écrit, couché sur le texte, côtoie l'image et sa disposition sur l'espace graphique est elle-même source d'iconicité. L'appropriation amérindienne de l'écriture alphabétique révèle que le symbole écrit peut être la trace d'une image dont il hérite parfois d'une siǵnification. De même, l'emblème et le «résumé hiéroǵlyphique» reproduits dans les livres de maǵie des traditions hermétiques ou alchimiques recèlent une dimension narrative secrète permettant à l'initié d'opérer la «primitive synthèse» pour accéder au savoir originaire (Giordana Charuty, ce volume). La perméabilité graphique ou la réversibilité du symbole et de l'icône sont présentes dans de nombreux usages rituels de l'écriture, depuis les traditions les plus anciennes des arts de la mémoire jusqu'aux manifestations plus récentes des arts de la parole associés à l'image (Yates 1966; Carruthers 2002; Schmitt 2002; Severi 2007; Cesarino 2016; Guillaume-Pey 2016). L'écriture maǵique, en tant qu'acte, possède sa dynamique interne de conversion des signes. Elle agit par figures, changements, inversions, coupures, opérés sur les noms des vivants, des morts ou des dieux qui se trouvent inscrits en position contiguë. Elle donne lieu à une interprétation matérielle du signe et s'inscrit dans un système de dérivation incluant la circulation d'objets rituels pouvant se substituer aux textes eux-mêmes, comme les arts de la mémoire, memoria verborum et memoria rerum. L'écrit magique acquiert une valeur rituelle d'échange entre les initiés et les dieux. Il s'inserit alors dans un dispositif de transmission dans lequel il circule en tant qu'artefact, support de texte, parmi d'autres objets rituels (Andrea-Luz Gutierrez-Choquevilca, ce volume; Hugh Jones et Diemberger 2012; Huǵh-Jones 2010).

Une autre forme de réflexivité se réfère au texte lui-même en tant qu'ensemble ordonné en séquences de siǵnes, dont la configuration est à chaque fois unique
2. Voir l'œuvre Les Yeux goinfres: les mangeurs de textes (1944 Tunisie) 12 dyptiques, série «Machines à voir» 1992, Centre Pompidou, Musée national d'art moderne/ Centre de création industrielle: https://www.centrepompidou. $\mathrm{fr} / \mathrm{fr} /$ ressources/oeuvre/ cKaAopn. 


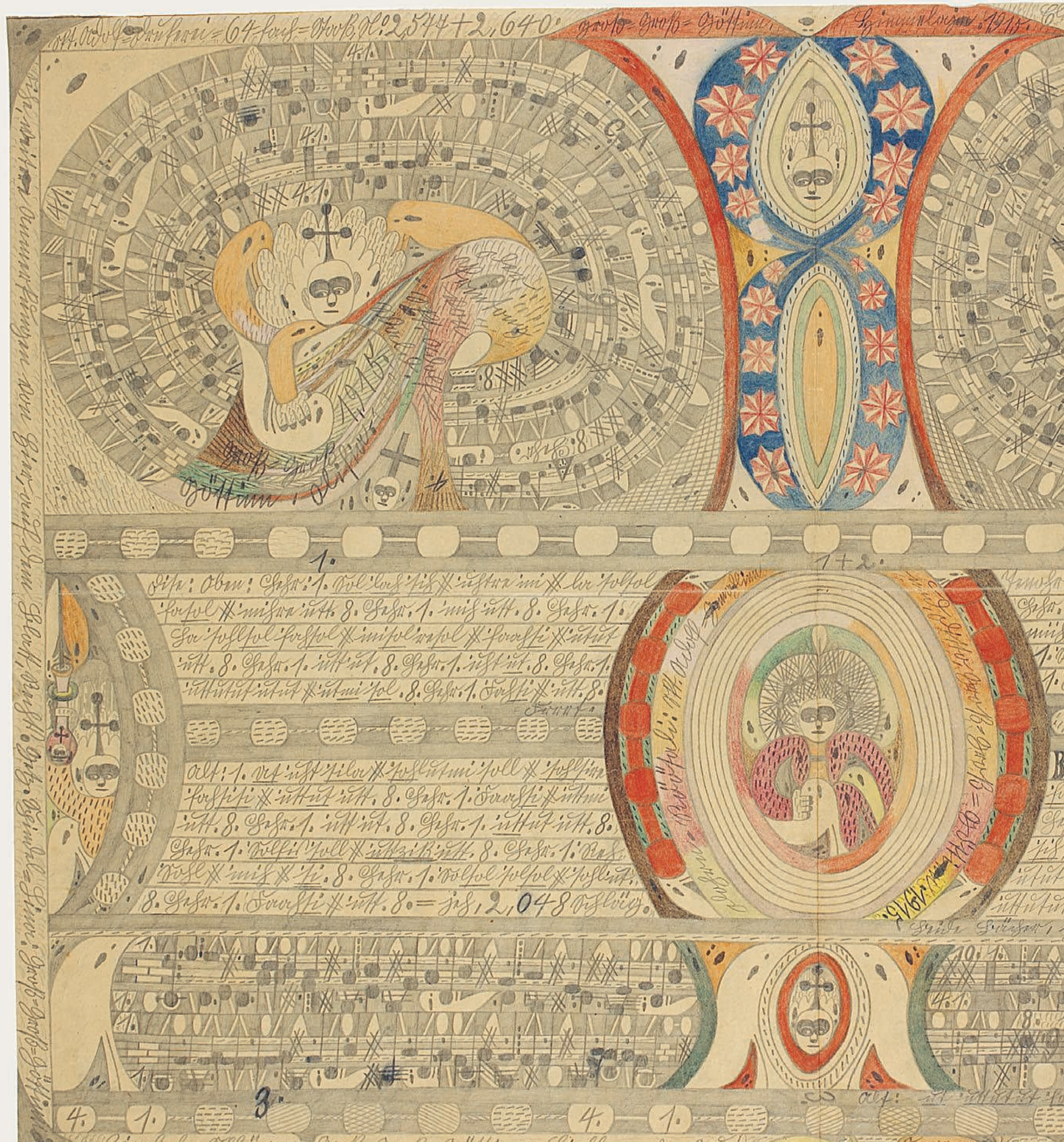




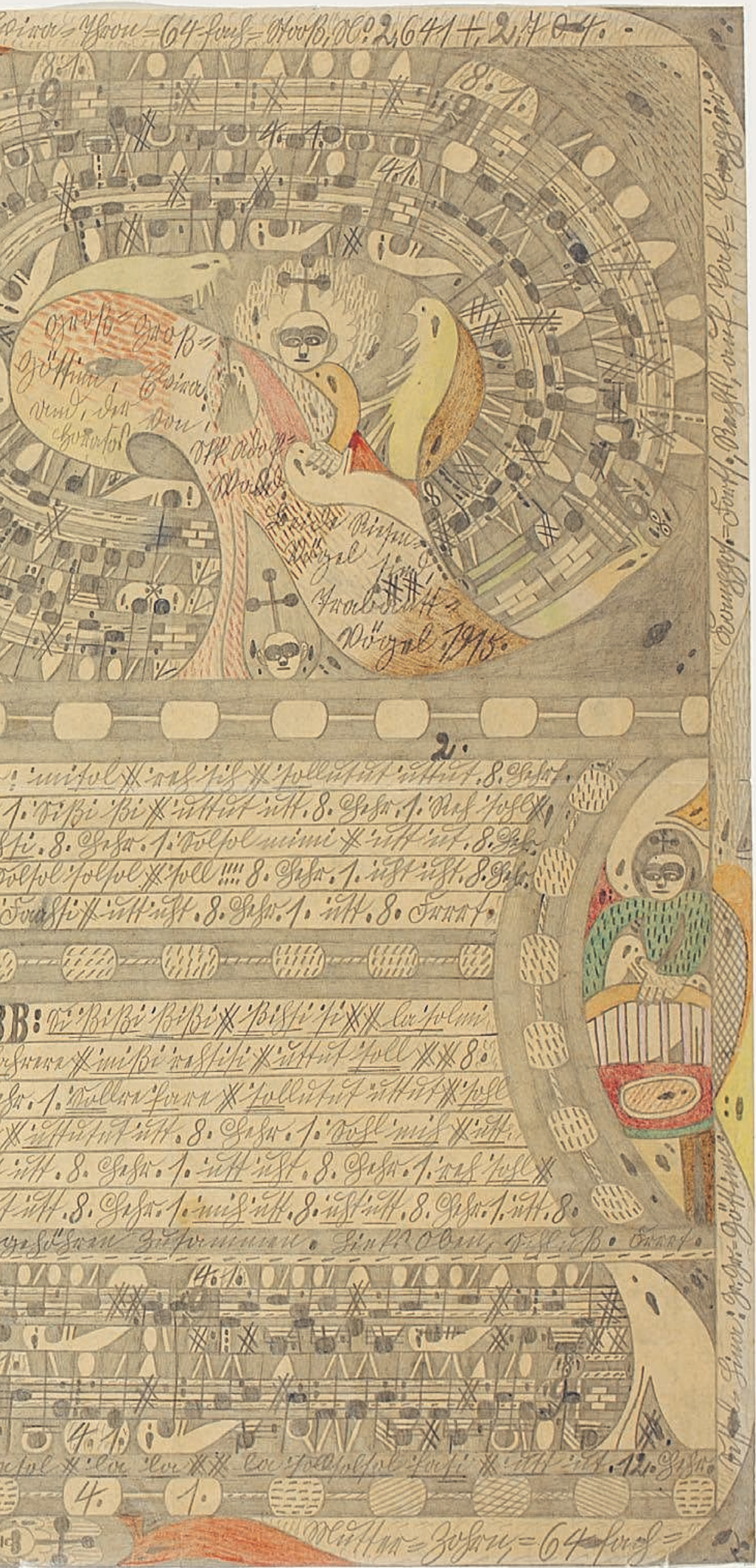

Adolf Wölfli, Les deux oiseaux géants de Saint Adolf, 1915 Texte, dessin, mine de plomb, crayon de couleur,

$72,5 \times 100 \mathrm{~cm}$.

Photo: Claude Bornand. Collection de l'Art Brut, Lausanne. Numéro d'entrée: cab-921. 
3. L'utilité de ce concept pour l'étude des écritures rituelles est heuristique. II invite à porter notre regard sur tout ce qui metle texte en relation, manifeste ou secrète, avec un autre texte, ou avec une réalité extratextuelle, dont il retire une forme, une force et une signification nouvelle et dont il porte la trace.

4. Voir Havelock 1981; Goody 1979; Olson et Torrance 1991. mais dont la forme peut donner lieu à des répétitions ou des variations internes ou externes, en principe infinies. De nombreuses ethnographies proposées dans ce volume présentent les corpus de textes étudiés comme des systèmes de transformations. Ce niveau de relation entre les signes relève d'un phénomène que les théoriciens de l'analyse littéraire qualifient de «transcendance textuelle ${ }^{3}$ » (Genette 1982).

La structure de significations que l'écrit véhicule n'est pas immuable, mais divisée en autant de niveaux de lectures qu'il y a de lecteurs, d'auteurs ou de scribes possibles. On mesure alors la distance ou au contraire l'étroite contiguiité reliant le texte, l'hypertexte à un hypotexte d'oriǵine, qui reste parfois lui-même introuvable. Prenons l'exemple des écritures nocturnes mayas cruzo'ob dévoilées par l'enquête de Valentina Vapnarsky et de Hilario Chi Canul dans le Quintana Roo, au Mexique. Voici des carnets palimpsestes dans lesquels se côtoient les textes de plusieurs auteurs (associés à des dates allant de 1904 à 1970), alliant des genres discursifs hétérogènes : prières, récits de la passion, regiistres de comptes et de décès, énoncés historiques, contes exemplaires, etc. Ils ont été transmis par un homme qui en iǵnore le contenu. Le siǵne est devenu presque illisible car écrit dans une lanǵue obscure rédigée en maya et en latin. La possession de ces carnets déclenche chez Hilario un rêve dans lequel apparaît l'esprit de l'ancien maître des carnets : ainsi naît dans l'obscurité, le désir de connaissance qui mène Hilario et Valentina sur les traces des auteurs secrets de ces liǵnes. L'analyse de la matière, du contenu et des cheminements des carnets au fil des mains mayas, de ceux qui les ont écrits, oralisés ou étudiés - scribes, prieurs, sorciers, politiciens, anthropologues - permet de reconstruire de la biographie de ces textes et de leurs manipulateurs. Une caractéristique des écrits magiques apparaît: la copie générative et créatrice de nouveaux textes. Composés d'assemblaǵes textuels, les écrits nocturnes se caractérisent par une hybridité interne, portant la trace de multiples auteurs qui se sont succédé dans le temps. L'écrit nocturne maya est dans son essence même une réécriture. L'intertextualité se fonde sur d'importantes répétitions externes: des fraǵments de textes sont reproduits sans jamais coïncider parfaitement avec l'original. L'acte de «copier» ne trouve précisément d'autre nom en maya que celui de «rénovation» ou de «renouvellement». S'il possède une valeur mnémonique pour le scribe alphabétisé, celle-ci n'épuise en rien les virtualités du texte. L'efficacité de la parole rituelle est étroitement liée à ce dispositif de variation qui vient ancrer la performance du tracé dans un hic et nunc propre, individualisant le rapport de l'orant à sa production. Chez les chamanes écrivains d'Amazonie, comme chez les Mayas du Yucatán, l'acte d'écrire se caractérise par un processus de réduplication infini. Une écriture rituelle en appelle une autre, sans discontinuer, s'accompaǵnant d'infimes variations.

Loin d'atténuer l'efficacité du livre rituel, sa traduction et sa copie présentent un caractère nécessaire, au point que la version originale pourrait presque paraître insignifiante au regard des variantes qu'une tentative de reconstitution exhaustive de la mémoire orale pourrait engendrer. L'exemple du livre de Xocén étudié par Pierre Déléage est exemplaire. Ironiquement, le livre des livres en vient à cumuler successivement le statut d'authentique exemplaire disparu, de copie puis de véritable «faux» livre, évoquant sans doute à l'esprit du lecteur la bibliothèque imaginaire de Babel citée en incipit. L'auteur introduit le lecteur dans le récit haletant d'un vol et d'une disparition du livre sacré, symbole de la perte d'autonomie et de la dépossession du savoir traditionnel maya sous la violence colonisatrice. Au fil des réécritures successives, le livre fait l'objet d'une reconquête par les habitants de Xocén. Le savoir consignné dans le livre retrouvé acquiert alors une dimension prophétique. Il révèle une «théorie maya de l'histoire» fondée sur l'interprétation de l'avènement de la société moderne, depuis ses infrastructures de communication jusqu'à son économie capitaliste et guerrière. Caractéristique d'une forme de transmission fondée sur la révélation, le savoir consignné dans les paǵes de ce livre est envisagé par les Mayas de Xocén comme toujours déjà là, donné, dans sa finitude et sa totalité, dans un unique Livre, contenant l'intégralité de ce qui a été dit et de ce qui adviendra. Le livre de Xocén rejoint ainsi l'utopie du «livre total» borgésien.

\section{L'ÉGRITURE GÉNÉRATIVE}

La poétique du palimpseste révèle les mécanismes de variations du savoir rituel et le lien indissoluble que ce dernier tisse avec l'oralité. Sur le plan cognitif, la production de l'écrit maǵique emprunte un schéma génératif, qui n'est pas étranger à ce que Jack Goody décrit comme une propriété typique de la mémoire orale, donnant lieu à la création de versions multiples d'un texte indépendant et constamment remanié (Goody 1977 : 40). Il va sans dire que la fixation immuable d'un texte et le détachement de la figure d'un auteur originel ne constituent pas l'objectif principal de ces écritures rituelles. Comme les arts de la mémoire qui coexistaient avec l'écriture en Europe et dans l'Antiquité, l'usage maǵique ou rituel de l'écrit coexiste lui aussi, comme en témoignent les études réunies dans ce volume, avec un usage administratif ou ordinaire de l'écriture, répondant à un besoin humain de remémoration et de stockage de l'information. L'écrit magique se développe parallèlement et répond à un tout autre besoin : celui d'articuler la mémoire et la genèse d'un signne matérialisé par l'acte d'inscription avec une séquence de gestes et de discours rituels. Ces écritures «attachées» à un contexte rituel qui en garantit la transmission remplissent alors des fonctions sociales multiples, allant de la fondation du charisme ou de l'autorité à des fins politiques ou religieuses à la fonction performative de l'acte de langage au sens qu'elle revêt chez John L. Austin (Déléage 2013 : 12). Plutôt que d'impliquer un saut cognitif majeur comme le présume l'hypothèse de la literacy ${ }^{4}$, l'appropriation magique de l'écriture alphabétique se voit structurellement 
Palimpseste, l'écriture rituelle au second degré

Andrea-Luz Gutierrez-Choquevilca

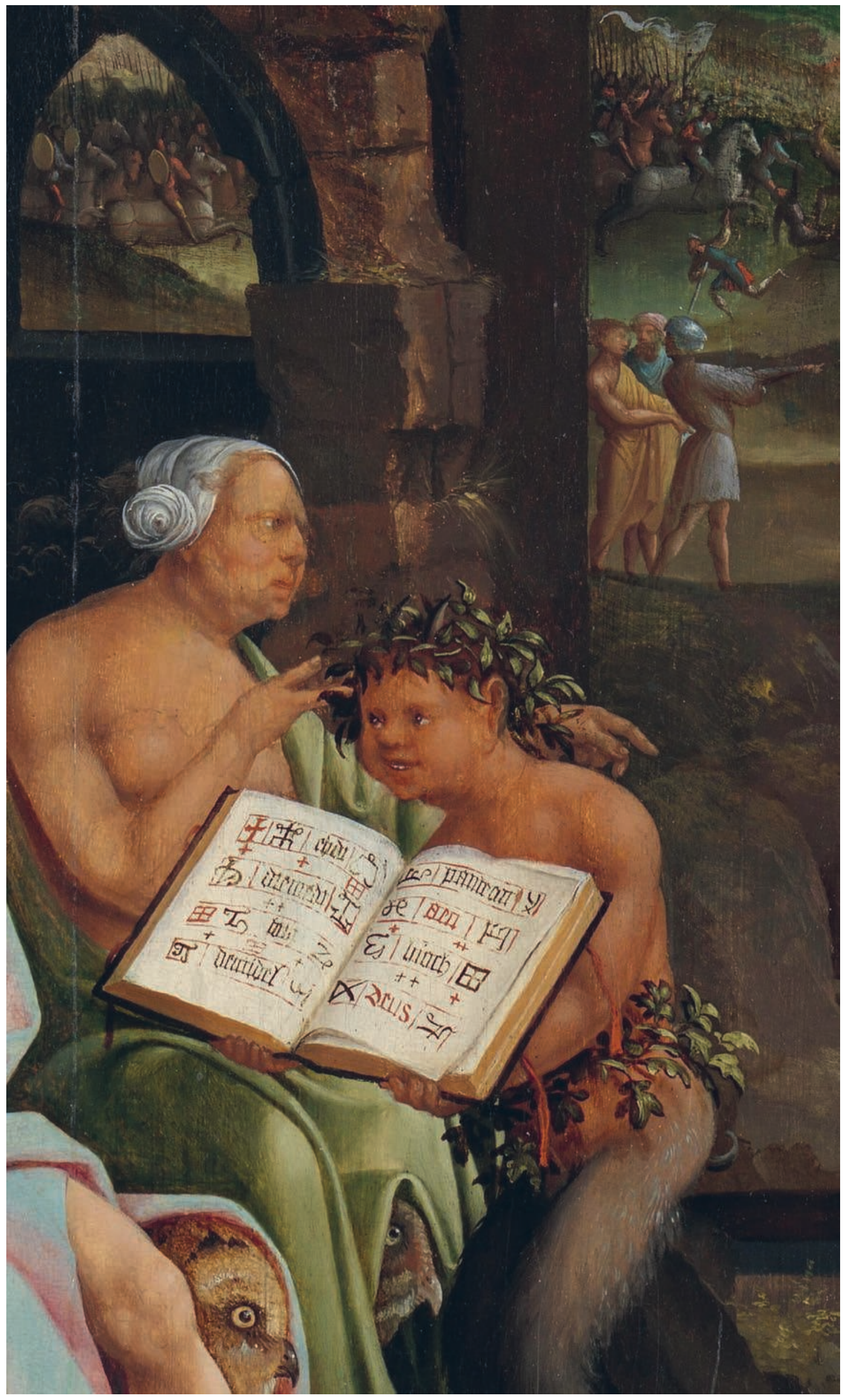

Jacob Cornelisz van Oostsanen, Saül et la Sorcière d'Endor (détail), 1526. Huile sur toile, 85,5 × 122,8 cm. Rijksmuseum, Amsterdam, inv. SK-A-668. 


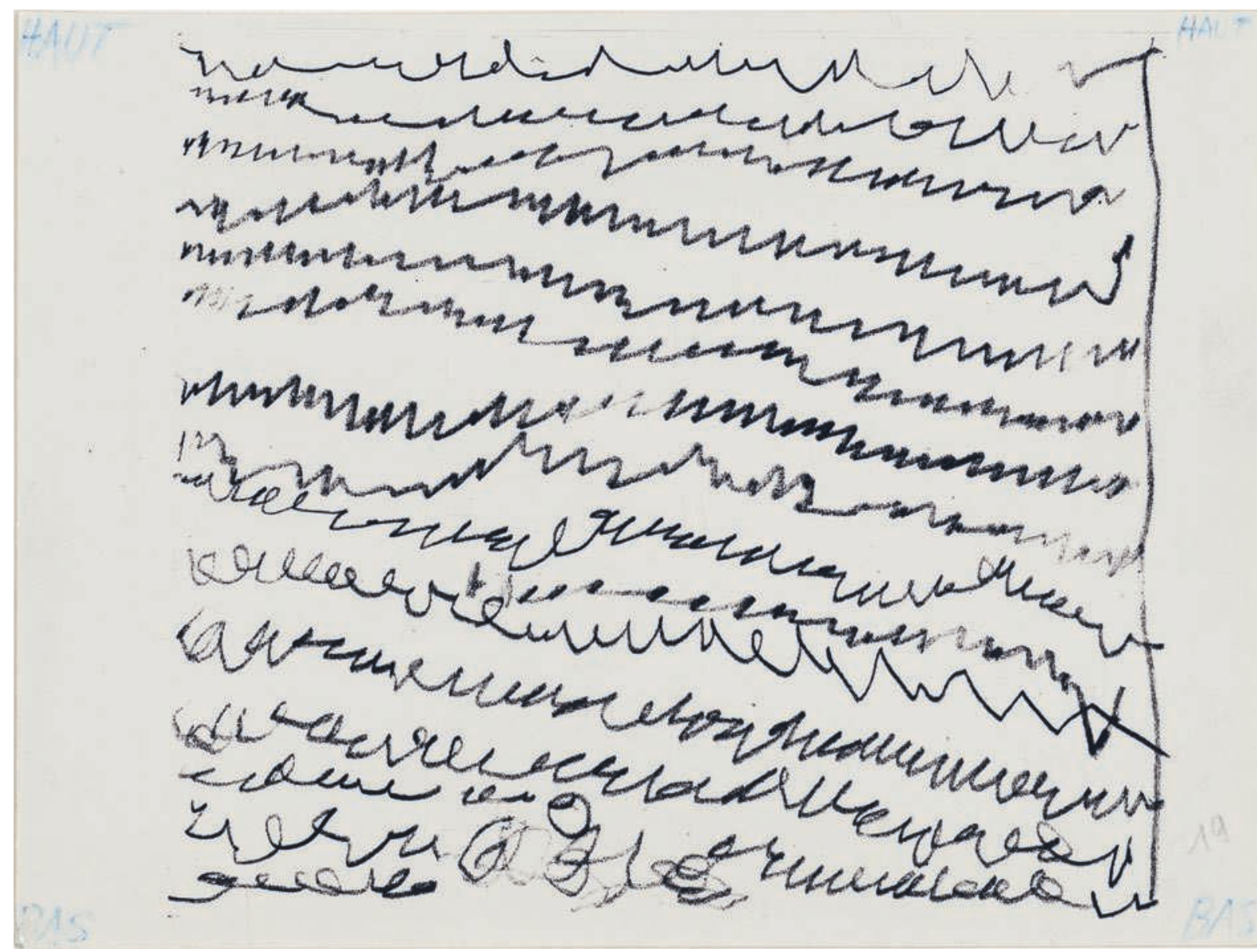

Page d'écriture d'un Indien Nambikwara, Claude Lévi-Strauss, Carnets Nambikwara, 1964 Paris, Bibliothèque nationale de France (BnF), NAF 28150 (24).

affectée par le régime de mémoire et d'imagination qui l'accueille en son sein. Parmi les peuples où existe déjà un type de remémoration générative favorable aux variations, l'usage rituel de l'écriture alphabétique par des maîtres de la parole lettrés s'insère dans une épistémologie et une praǵmatique typiques de l'oralité Il en résulte que la distance qui séparait le prophète analphabète incapable de lire le livre autrement que virtuellement et le chamane écrivain lettré capable de lire littéralement, mais non satisfait de se réduire à cette modalité de saisie du symbole linguistique, devient ténue voire inconsistante (Gow, 2001; Gutierrez-Choquevilca, ce volume; Déléage 2013; Névot 2011). Ce n'est pas l'aptitude à lire ou à écrire, mais cette herméneutique variable du signe qui définit le caractère "spécial» de l'écriture magique.

D'où vient l'efficacité magique attribuée au symbole écrit? Elle découle d'une construction singulière de l'autorité religieuse. Il faut en revenir à l'épisode marquant de la transmission. C'est la relation entre un scripteur et un auteur invisible, magnifié, déifié, canonisé, qui fonde l'autorité et la puissance attribuées à ces textes. L'épistémologie des écritures inspirées repose sur la révélation d'un savoir à l'initié. Ce savoir révélé constitue un hypotexte rituel, combinant un usaǵe étendu des symboles, des indices ou des icônes. Une série de paroles sont énoncées lors d'un rêve ou d'une vision. Des incantations secrètes sont entendues dans un état altéré de la conscience. Le cas des écritures inspirées d'Amazonie révèle ainsi que pour devenir écrivain, le chamane amérindien doit être aussi lecteur et interprétant du signe, quand bien même cette lecture reste purement virtuelle. C'est au cours d'une vision que le chamane runa perçoit simultanément les «lettres créatures » des esprits supay et leurs chants, qu'il consignera le lendemain sur papier (Andrea-Luz Gutierrez-Choquevilca, ce volume). La particularité de cette lecture imaǵinaire est de se fonder sur un principe de permutation sémiotique : la possibilité simultanée de «lire» un même siǵne sous des modalités variables, de percevoir la transformation subtile d'une image figurative en signe visuel tracé, puis en texte, par un jeu de cryptage et de décryptaǵe du savoir. L'écriture serait ainsi la manifestation tangible d'une voix révélée, celle de Dieu ou des esprits, sans qu'aucun canal sémiotique exclusif ne parvienne à épuiser la signnification du savoir ainsi transmis. La dissociation possible de la figure du scribe de celle de l'auteur met en évidence la distance qui sépare et relie la production du texte à une forme d'autorité dont l'écrit porte la trace diffuse. Il apparaît clairement que ce qui est produit ici n'est pas exclusivement un savoir, mais une chaîne de transmission. Cette chaîne fait intervenir de multiples acteurs, parmi lesquels figurent un énonciateur idéal, un témoin visionnaire, un scribe et un expert rituel, ces dernières fonctions pouvant être cumulées par une même personne. L'exemple du prophétisme scripturaire africain, reproduisant les actes administratifs et la bureaucratie coloniale, 
étudié par Julien Bonhomme, en dévoile l'une des manifestations possibles: la signature du prophète Kimbangu, dont les «passeports pour le ciel » portent la trace, attestant de la validité du savoir couché sur la page, de l'autorité prêtée au texte et partant au détenteur de l'écrit. Technologie de communication à distance, l'écrit est un médium adapté à ce mode singulier d'existence: le messie est physiquement absent, mais sa signature et ses lettres sont autant de traces indicielles de sa présence.

\section{ÉCRITURES SUBVERSIVES}

La stabilisation des écritures rituelles dans le temps s'articule avec le développement d'institutions, de sociétés religieuses et de pratiques s'inscrivant parfois à contre-courant du pouvoir dominant. La problématique d'une corrélation hypothétique entre la diffusion de l'écriture et l'avènement de l'État n'est pas l'enjeu de la réflexion menée dans ce volume. Néanmoins plusieurs contributions interrogent ici avec finesse la façon dont l'écriture vient s'articuler dans des rapports sociaux qui en déterminent le sens, la valeur et les effets. Le pouvoir conféré à l'écriture rituelle ne peut être envisagé in abstracto, car il dépend des contextes sociohistoriques dans lesquels les documents sont produits et circulent. Ainsi l'appropriation des livres de magie dans la paraphernalia et les pratiques du chamanisme amazonien décrites par Pierre Déléage s'inscrit-elle dans un processus de mise en concurrence des savoirs au niveau local. La circulation secrète des grimoires de maǵie et du livre de San Cipriano, issus de l'Ancien Monde, vient subvertir un ordre existant et se substituer temporairement à d'autres objets et supports de connaissances, tels que les plantes, substances et vecteurs classiques de la transmission du savoir chamanique en Amazonie (Déléage, ce volume). Ce transfert n'est pas étranger à la logique prévalant au sein des traditions chamaniques, qui accordent un pouvoir exceptionnel aux technologies venues d'ailleurs et plus généralement à toute forme de savoir susceptible de concurrencer, dans une logique de combat entre les forces invisibles, l'autorité et la puissance des savoirs locaux (Taylor 1981; Deshoullière 2016). L'appropriation amérindienne des livres de maǵie s'inscrit dans un conflit générationnel, reflet d'un désir d'émancipation au sein d'une tradition régionale établie.

La circulation secrète des textes maǵiques peut être la manifestation d'un pouvoir subversif qui se développe en marǵe des systèmes religieux dominants, dans le cadre de la concurrence entre plusieurs institutions politiques et religieuses. L'analyse des formes bureaucratiques du prophétisme en contexte colonial en offre une illustration parfaite. Si les sujets colonisés ont fait l'expérience du pouvoir de l'écrit par le biais de la Bible, mais aussi à travers pléthore de documents administratifs - titres de propriété, livrets d'identité, reçus d'impôt, procès-verbaux de police -, c'est à travers un type spécifique d'écriture révélée, les «passeports pour le ciel», que s'opère une institutionnalisation des mouvements prophétiques caractérisés par un anticolonialisme affiché aux accents millénaristes. Ce qui est en jeu n'est pas l'écriture en tant que telle et son rôle de technologie cognitive suppléant à la mémoire humaine, mais sa vocation politique et religieuse d'émancipation à l'égard des Européens. Ces signnes écrits ont l'avantage d'être manipulables par autrui, la dissociation entre le scripteur et l'auteur permettant de faire parler le prophète Kimbangu à la première personne. L'auctorialité de l'écrit constitue ainsi une modalité importante de la construction d'une autorité messianique. L'appropriation reliǵieuse des papiers d'identité passe par un renversement symbolique typique du messianisme. L'opérateur qui rend possible cette subversion, c'est «Kimbanǵu, Roi $\mathrm{du}$ Congo » : non pas la personne réelle, mais la figure symbolique du Messie que les adeptes opposent au pouvoir colonial en lui faisant prendre à la fois la place du Christ et celle du gouverneur général. C'est à la force de cette bureaucratie céleste que revient le pouvoir de renverser l'ordre colonial pour établir un nouvel ordre politique et religieux.

Les contributions réunies dans ce volume ne visent pas à épuiser les interrogations portant sur le lien entre écriture et oralité, pas plus qu'elles n'apportent de réponse uniforme à la question de l'origine du signe magique. Plus modestement, ces réflexions inviteront le lecteur à explorer l'épistémologie des écritures rituelles par un retour au commencement. Pour insaisissable qu'il soit, comme le livre de sable borgésien, celui-ci est à chercher dans la révélation d'un monde invisible où se joue la conversion possible, infinie, du signe écrit en parole vive, en image et en index d'une présence.

École pratique des hautes études PSL,

Laboratoire d'anthropologie sociale andrea-luz.gutierrez-choquevilca@college-de-france.fr 


\section{Bibliographie}

Borges, Jorge Luis

1983 Fictions, trad, de l'espaǵnol par P. Verdevoye, Ibarra et Roger Caillois.

Paris, Gallimard.

1978 Le Livre de sable, trad. revue par Jean-Pierre Bernes. Paris, Gallimard.

\section{Carruthers, Mary}

2002 Machina Memorialis méditation, rhétorique et fabrication des images au Moyen Âge, trad de l'anǵlais par Fabienne Durand-Bogaert.

Paris, Gallimard.

\section{Cesarino, Pedro N.}

2016 «Cartographies du cosmos. Image, parole et savoir chez les Marubo », in Carlos Fausto et Carlo Severi (dir.), Paroles en images, écritures, corps, mémoire. Marseille,

OpenEdition Press.

\section{Davies, Owen}

2009 Grimoires: A History of Magic Books. Oxford, OUP Oxford.

\section{Déléage, Pierre}

et Morin, Olivier

2018 "Écritures recyclées», Terrain 70 [en liǵne],

disponible sur: http://journals. openedition.orǵ/terrain/17046 DOI : https://doi.org/10.4000/ terrain (consulté le 12 février 2021).

\section{Déléage, Pierre}

2013 Inventer l'écriture. Paris, Les Belles Lettres.

2017 Lettres mortes : essai d'anthropologie inversée. Paris, Fayard.

\section{Deshoullière, Grégory}

2016 «Vanités aux livres. Variations maǵiques en pays jivaro », Hypalampuses hemeras - A la pointe du jour (Revue Gruppen) : 42-55.

\section{Fraenkel, Béatrice}

1992 La signature : genèse d'un signe. Paris, Gallimard.

2006 "Actes écrits, actes

oraux. La performativité à

l'heure de l'écriture », Études

de communication 29: 69-93.

\section{Genette, Gérard}

1982 Palimpseste : la littérature au second deǵré. Paris, Seuil.

\section{Glassner, Jean-Jacques}

2008 «e corps écrit.

La victime dans le sacrifice divinatoire en Mésopotamie», in Brigitte Baptandier et Giordana Charuty (éd.), Du corps au texte. Nanterre, Société d'ethnoloǵie: 185-206.

\section{Goody, Jack}

1977 «Mémoire et apprentissage dans les sociétés avec et sans écriture. La transmission du Baǵre», L'Homme 17 (1) 29-52.

1979 La Raison graphique: la domestication de la pensée sauvage, trad. de l'anólais par Jean Bazin et Alban Bensa. Paris, Minuit.

\section{Gow, Peter}

2001 An Amazonian Myth and Its History. Oxford Oxford University Press.

\section{Guillaume Pey, Cécile}

2016 «Boire des lettres ou converser avec les esprits? Tensions rituelles autour de l'écrit chez les Sora Ethnographiques.org 33

Ethographicues.org 33 https://www.ethnographiques. https://www.ethnographiqu (consulté le 12 février 2021)

\section{Havelock, Eric A.}

1981 Aux origines de la civilisation écrite en Occident, trad. de l'anǵlais par E. Escobar Moreno. Paris, Maspero.

\section{Hugh-Jones, Stephen}

2010 «Entre l'imaǵe

et l'écrit: la politique tukano de patrimonialisation en Amazonie ", Cahiers des Amériques latine 63-64: 195-228.

Hugh Jones, Stephen et Diemberger, Hildegard 2012 «L'objet-livre», Terrain 59 [en ligne], disponible sur : https://journals.openedition org/terrain/14877\#entries DOI : https://doi.org/10.4000/ terrain.14 (consulté le 12 février 2021)

Koch Piettre, Renée et Batsch, Christophe (dir.) 2010 Cahiers des mondes anciens 1, «Écritures rituelles».

\section{Névot, Aurélie}

2011 «Une écriture secrète sur la scène publique en Chine», Revue de l'histoire des relioions 2: 227-243.

\section{Olson, David et Torrance,} Nancy (dir.)

1991 Literacy and orality. New York, Cambridge University Press.

\section{Rendu-Loisel, Anne-Caroline} 2018 «Le prêtre incantateur est-il un scribe raté? Incantations et langagóes efficaces dans l'ancienne Mésopotamie», Parcours anthropologiques 13 : 94-109.

\section{Schmitt, Jean-Claude}

2002 Le corps des images : essais sur la culture visuelle au Moyen Âge. Paris, Gallimard.

Taylor, Anne-Christine

1981 «God-Wealth: The Achuar and the Missions », in Norman E. Whitten (dir.), Cultural Transformations and Ethnicity in Modern Ecuador. Urbana, University of Illinois Press : 647-676.

\section{Wogan, Peter}

1994 «Perceptions of

European Literacy in Early Contact Situations », Ethnohistory 41 (3) : 407-429.

\section{Yates, Frances A.}

1966 The Art of Memory. Chicago/Londres, University of Chicago Press/Routledge and Kegan Paul.

\section{Severi, Carlo}

2007 Le Principe de la chimère: une anthropologie de la mémoire. Paris, Rue d'Ulm/Musée du quai Branly.

\section{Ci-contre et ouverture}

Page d'écriture d'un Indien Nambikwara, Claude Lévi-Strauss, Carnets Nambikwara, 1964 (détail). Paris, Bibliothèque nationale de France (BnF), NAF 28150 (24). 


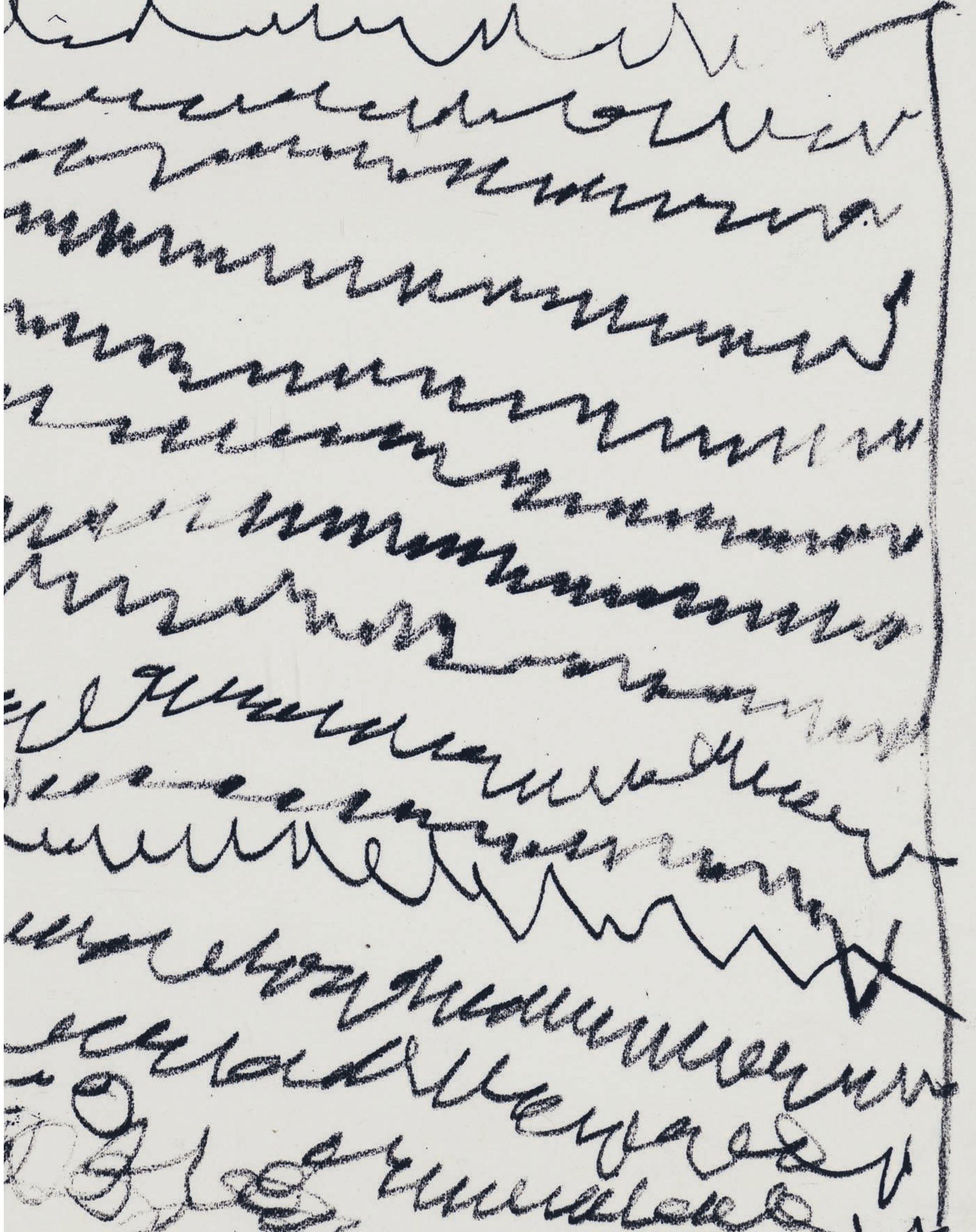

